\title{
Stent sizing strategies in renal artery stenting: the comparison of conventional invasive renal angiography with renal computed tomographic angiography
}

\author{
Jacek Kadziela ${ }^{1}$, llona Michalowska ${ }^{2}$, Jerzy Pregowski ${ }^{1}$, Hanna Janaszek-Sitkowska ${ }^{3}$, Katarzyna Lech ${ }^{1}$, \\ Marek Kabat ${ }^{3}$, Adam Staruch ${ }^{1}$, Andrzej Januszewicz ${ }^{1}$, Adam Witkowski ${ }^{1}$ \\ ${ }^{1}$ Department of Invasive Cardiology and Angiology, Institute of Cardiology, Warsaw, Poland \\ ${ }^{2}$ Department of Radiology, Institute of Cardiology, Warsaw, Poland \\ ${ }^{3}$ Department of Hypertension, Institute of Cardiology, Warsaw, Poland
}

Adv Interv Cardiol 2016; 12, 2 (44): 116-121

DOI: 10.5114 /aic.2016.59361

\begin{abstract}
A bstract
Introduction: Randomized trials comparing invasive treatment of renal artery stenosis with standard pharmacotherapy did not show substantial benefit from revascularization. One of the potential reasons for that may be suboptimal procedure technique.

Aim: To compare renal stent sizing using two modalities: three-dimensional renal computed tomography angiography (CTA) versus conventional angiography.

Material and methods: Forty patients (41 renal arteries), aged $65.1 \pm 8.5$ years, who underwent renal artery stenting with preprocedural CTA performed within 6 months, were retrospectively analyzed. In CTA analysis, reference diameter (CTA-D) and lesion length (CTA_LL) were measured and proposed stent diameter and length were recorded. Similarly, angiographic reference diameter (ANGIO_D) and lesion length (ANGIO_LL) as well as proposed stent dimensions were obtained by visual estimation.

Results: The median CTA_D was $0.5 \mathrm{~mm}$ larger than the median ANGIO_D $(p<0.001)$. Also, the proposed stent diameter in CTA evaluation was $0.5 \mathrm{~mm}$ larger than that in angiography $(p<0.0001)$. The median CTA_LL was $1 \mathrm{~mm}$ longer than the ANGIO_LL $(p=N S)$, with significant correlation of these variables $(r=0.66, p<0.0001)$. The median proposed stent length with CTA was equal to that proposed with angiography. The median diameter of the implanted stent was $0.5 \mathrm{~mm}$ smaller than that proposed in CTA $(p<0.0005)$ and identical to that proposed in angiography. The median length of the actual stent was longer than that proposed in angiography $(p=0.0001)$.

Conclusions: Renal CTA has potential advantages as a tool adjunctive to angiography in appropriate stent sizing. Careful evaluation of the available CTA scans may be beneficial and should be considered prior to the planned procedure.
\end{abstract}

Key words: renal artery stenosis, renal artery stenting, computed tomographic angiography, conventional angiography, renovascular hypertension.

\section{Introduction}

Percutaneous angioplasty with stent implantation may be considered a viable therapy in highly selected patients with renal artery stenosis [1-4]. Randomized trials comparing invasive treatment with standard pharmacotherapy did not show substantial benefit from revascularization of hypertensive patients [5-7]. One of the potential reasons for the apparent lack of benefit may be suboptimal procedure technique. Inadequate stent sizing may lead to stent malapposition or underexpansion and may result in a higher thrombosis rate, increased risk of in-stent restenosis and worse long-term prognosis.
Routinely, stent sizing is based on visual estimation of the stenosed artery during initial renal angiography. On the other hand, the majority of patients with suspected renovascular hypertension undergo renal computed tomographic angiography (CTA). The complementary assessment of the renal artery stenosis in CTA and proper evaluation of the plaque and the reference segment diameter may improve the accuracy of stent sizing.

\section{Aim}

The aim of this study was to compare stent sizing for renal artery stenosis interventions using two modalities:

\section{Corresponding author:}

Jacek Kadziela MD, PhD, Department of Invasive Cardiology and Angiology, Institute of Cardiology, 42 Alpejska St, 04-628 Warsaw, Poland, phone: +48 605298 959, e-mail: j.kadziela@ikard.pl

Received: 30.11.2015, accepted: 29.12.2015. 
three-dimensional renal CTA versus conventional renal angiography.

\section{Material and methods \\ Study population}

Eighty-one hypertensive patients underwent renal artery stenting for atherosclerotic stenosis between January 2011 and December 2014. Forty of them (aged 65.1 \pm 8.5 years, 25 males) with preprocedural CTA performed within a maximum of 6 months prior to stenting (42 \pm 24 days) were retrospectively analyzed. In one patient bilateral renal artery stenting was done, so in summary 41 arteries of 40 patients were included in the analysis.

\section{CTA protocol}

The CTA scans were performed using a dual-source scanner (Somatom Definition Flash, Siemens, Erlangen, Germany). The following scanning parameters were applied: detector collimation $-128 \times 0.6 \mathrm{~mm}$, gantry rotation time $-280 \mathrm{~ms}$, tube voltage $100-120 \mathrm{kV}$ depending on the patient's body mass. Contrast-enhanced scans were obtained in the arterial phase after intravenous administration of $70-100 \mathrm{ml}$ of contrast material $(350 \mathrm{mg} / \mathrm{ml})$ injected at a flow rate of $5 \mathrm{ml} / \mathrm{s}$ via the antecubital vein followed by a $30-\mathrm{ml}$ saline chaser. Image acquisition was initiated $7 \mathrm{~s}$ after reaching $100 \mathrm{HU}$ threshold enhancement within the region of interest located in the descending aorta.

Using automated software, two- and three-dimensional reconstructions were obtained and proximal (if possible) and distal reference diameters were measured manually. The CTA reference diameter (CTA_D) was calculated as the mean of the proximal and distal reference diameter (Figure 1). In cases of plaques involving the ostium (37 cases), the distal reference diameter was recorded as the CTA_D (Figure 2). Minimal lumen diameter was measured at the narrowest point of the lesion and the percent diameter stenosis (CTA_DS) was calculated automatically. The distance between the distal and proximal end of the lesion (or the artery ostium) was measured as the lesion length (CTA_LL - Figure 1).

Based on the CTA_D results, a blinded interventional cardiologist proposed the stent diameter that was chosen from the following commercially available stent diameters: 4.0, 4.5, 5.0, 5.5, 6.0, 6.5, 7.0 mm. Similarly, based of the CTA_LL measurements and assuming that the stent should cover at least $1 \mathrm{~mm}$ of distal healthy segment and should protrude at least $1 \mathrm{~mm}$ into the aorta, the proposed stent length was chosen from the following lengths: 12, 15, 18, 20 or $24 \mathrm{~mm}$.

All plaques were also classified according to the presence of calcium as: non-calcified, mildly calcified (calcium covering up to $1 / 3$ of the plaque area), moderately calcified (1/3-2/3 area of calcium) and severely calcified (more than $2 / 3$ of the plaque area).

\section{Angiographic analysis}

All angiographic recordings were retrospectively evaluated by the blinded experienced operator. Based on the visual estimation, the following parameters were recorded (using the same rules as in CTA assessment - Figures 1 and 2): the diameter of the reference artery segment (ANGIO_D), the lesion length (ANGIO_LL), the percent diameter stenosis (ANGIO_DS) and calcium presence. Similarly, the proposed stent dimensions were selected from the following diameters: 4.0, 4.5, 5.0, 5.5, 6.0, 6.5, $7.0 \mathrm{~mm}$ and the following lengths : 12, 15, 18, 20 or $24 \mathrm{~mm}$.
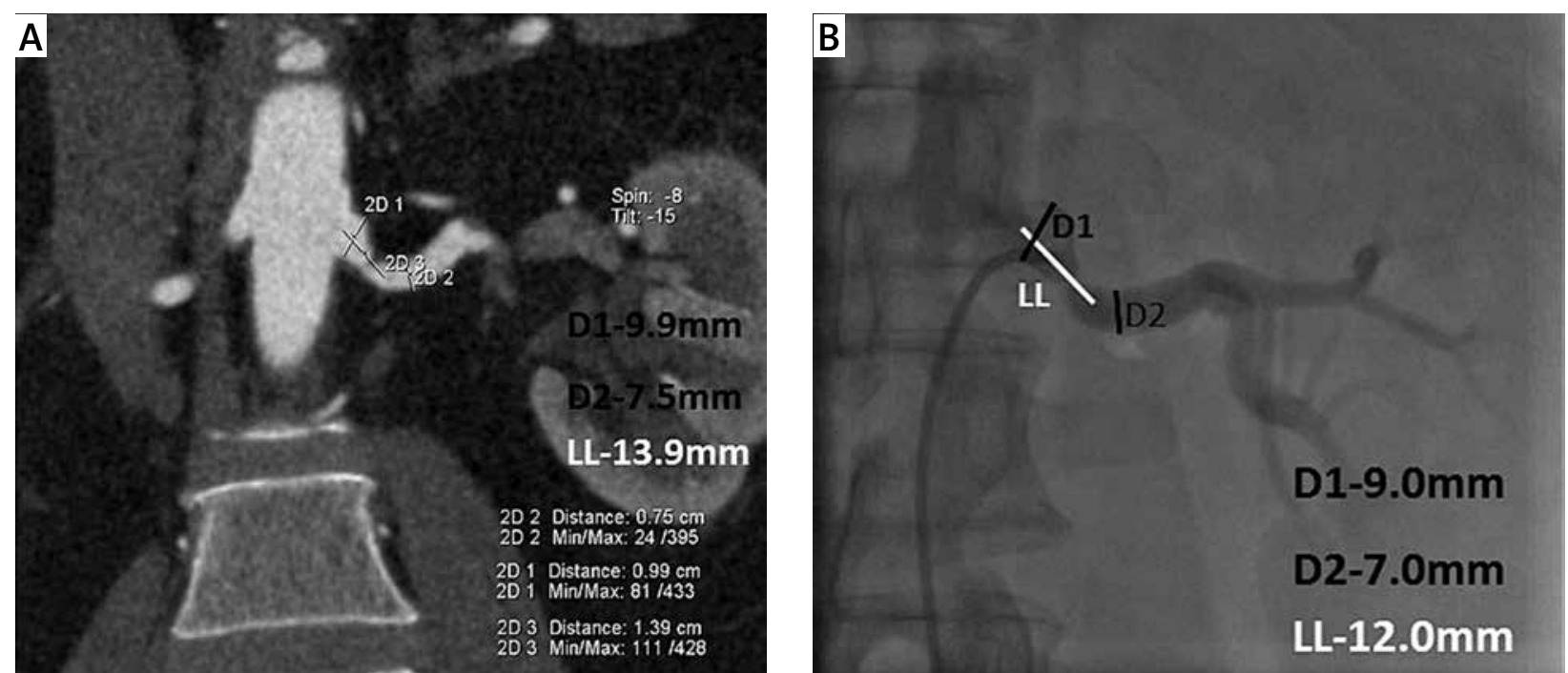

Figure 1. CTA (A) and X-ray angiography (B) of the left renal artery. Both proximal (D1) and distal (D2) reference diameters were measured. Reference diameter (CTA_D and ANGIO_D, respectively) was calculated as the mean

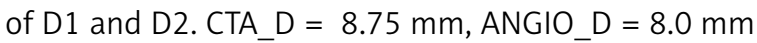

$L L$-lesion length. 

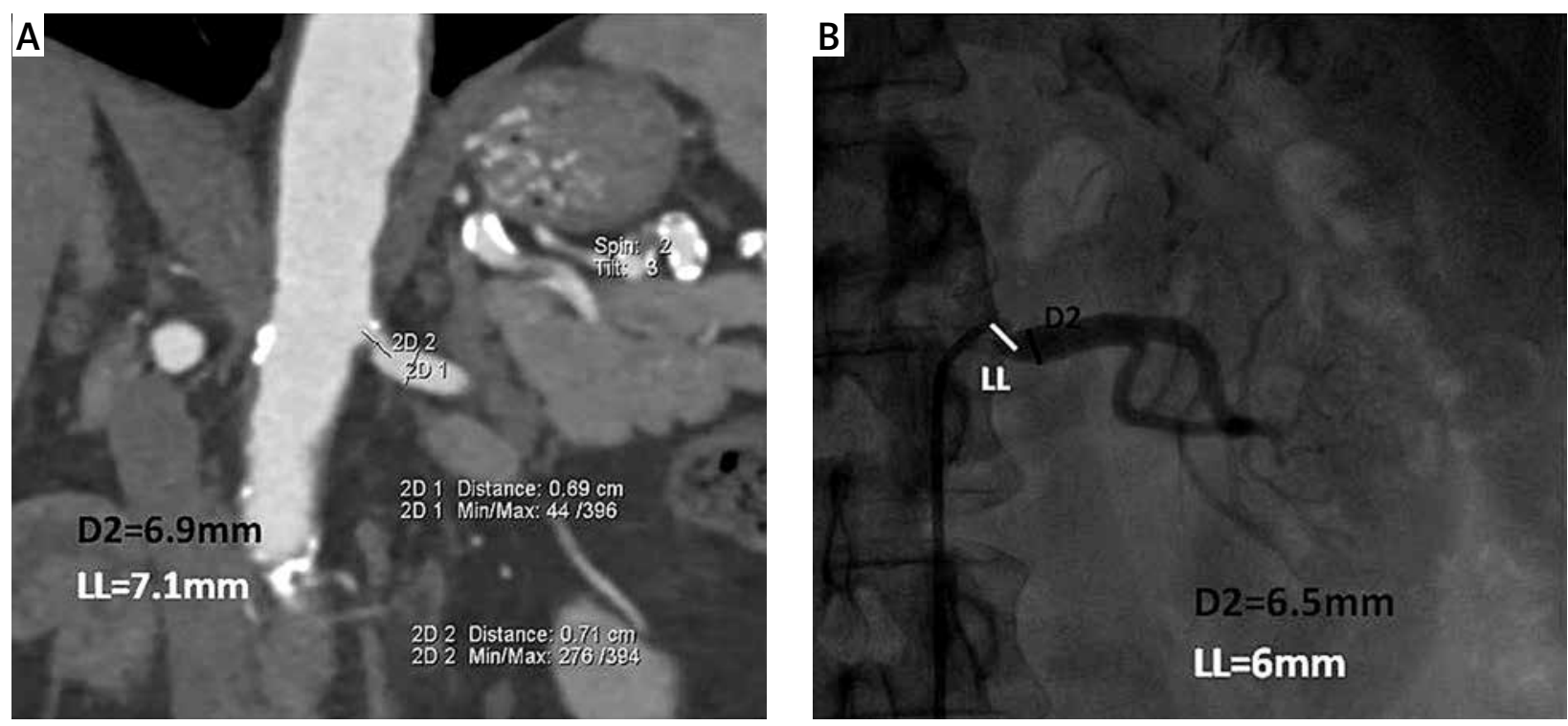

Figure 2. CTA (A) and X-ray angiography (B) of the left renal artery. Due to the ostial location of the lesion, proximal reference diameter (D1) was not measured. Distal reference diameter (D2) was recorded as the reference diameter. CTA_D $=6.9 \mathrm{~mm}, \mathrm{ANGIO} D=6.5 \mathrm{~mm}$

LL-lesion length.

\section{Renal artery stenting analysis}

Implanted stent dimensions were gathered as well as other procedure-related details including the name and manufacturer of the stent, deployment pressure, residual stenosis (RS), use of predilatation, predilatation balloon diameter, the need for postdilatation with a larger diameter balloon and final residual stenosis after postdilatation.

\section{Statistical analysis}

Continuous variables do not have a normal distribution and are presented as medians with interquartile ranges and were compared using the nonparametric Wilcoxon signedrank test. Categorical data are presented as frequencies. Two-tailed tests of significance are presented with $p$ value less than 0.05 considered statistically significant.

\section{Results}

\section{Lesion analysis}

The median CTA_D was $0.5 \mathrm{~mm}$ larger than median ANGIO_D $(p<0.001)$, as presented in Table I. Also, the median proposed stent diameter in CTA evaluation was $0.5 \mathrm{~mm}$ larger than that in angiography $(p<0.0001)$. The proposed stent diameters were identical in CTA and angiography only in 16 (39\%) cases.

The median CTA_LL was $1 \mathrm{~mm}$ longer than the median ANGIO_LL ( $p=N S)$, with a significant correlation found between these variables $(r=0.66, p<0.0001)$. The median proposed stent length in CTA was equal to that proposed in angiography.

The median diameter stenosis measured in angiography (85\%; interquartile range (IQR): 77.5-90) was higher than CTA-derived diameter stenosis (69.5\%; IQR: 62.1-76, $p<0.0001$ ). $36.6 \%$ of lesions in CTA and $58.5 \%$ in angiography were classified as non-calcified. $31.7 \%$ of lesions were categorized as moderately or severely calcified in CTA as compared with $9.8 \%$ in angiography.

\section{PTA analysis}

All patients underwent uncomplicated renal artery stenting - the characteristics of the procedure are depicted in Table II.

Table I. Results of computed tomographic angiography vs. conventional angiography analysis. Data presented as medians and interquartile ranges

\begin{tabular}{lcccc} 
Parameter & CTA & Angio & $P$-value & Implanted stent \\
\hline Reference diameter & $6.2(5.7-7.0)$ & $5.7(5.0-6.0)$ & $<0.0001$ & - \\
\hline Lesion length & $11.0(8.8-13.6)$ & $10(8.0-13.0)$ & NS & - \\
\hline Proposed stent diameter & $6.5(5.9-7.0)$ & $6.0(5.0-6.0)$ & $<0.0001$ & $6.0(5.0-6.0)^{*}$ \\
\hline Proposed stent length & $12(12-15)$ & $12(12-15)$ & NS & $15(12-15.75)^{\star *}$
\end{tabular}

${ }^{*} p<0.0005$ for comparison with proposed stent diameter in CTA, $p=$ NS for comparison with angiography. ${ }^{*} p=0.0001$ for comparison with proposed stent length in ANGIO, $p=$ NS for comparison with CTA. CTA - computed tomographic angiography, Angio - conventional invasive angiography, Implanted stent - stent dimensions used in renal artery stenting procedure. 
The median diameter and length of the implanted stent were $6.0 \mathrm{~mm}$ (IQR: 5.0-6.0) and $15.0 \mathrm{~mm}$ (IQR: 12.015.75), respectively. The median diameter of the implanted stent was $0.5 \mathrm{~mm}$ smaller than that proposed in CTA $(p<0.0005)$ and identical to that proposed in angiography (Table I). In 21 (51.2\%) cases the median diameter of the implanted stent was smaller than that proposed in CTA assessment, in 17 (41.5\%) cases it was equal and in $3(7.3 \%)$ patients it was higher than in CTA. The implanted stent diameter was consistent with the angiography proposal in $28(68.3 \%)$ patients, larger in $5(12.2 \%)$ and smaller in 8 (19.5\%) cases.

The median length proposed in CTA was equal to that proposed in angiography. However, the median length of the implanted stent was longer than retrospectively proposed in angiography $(p=0.0001)$. The implanted stent length was consistent with the CTA proposal in $16 \mathrm{pa}$ tients and with angiography in 22 patients.

The median residual stenosis (RS) after stent implantation was 10\% (IQR: 0-20\%). In 14 (34.1\%) patients RS was at least $20 \%$; postdilatation with a larger balloon was performed in only 3 of them. In this subgroup of 14 patients, the difference between the proposed stent diameter in CTA and the implanted stent diameter was significantly higher as compared to the subjects with residual stenosis less than $20 \%(1.0 \mathrm{~mm}$ (IQR: $0-1.5)$ vs. $0 \mathrm{~mm}$ (IQR: 0-0.875), $p<0.05)$.

\section{Discussion}

This study demonstrated the potential advantages of renal computed tomographic angiography as a tool adjunctive to angiography in appropriate stent sizing before renal artery stenting. The reference artery diameter and the proposed stent diameter based on CTA measurements were $0.5 \mathrm{~mm}$ larger than those proposed in angiography. Moreover, in patients with at least $20 \%$ residual stenosis in angiography, the discrepancy between CTA-selected and actually used stent diameter was more pronounced. Coronary studies confirmed that stent underexpansion and significant residual stenosis increase the risk of stent thrombosis and in-stent restenosis [8-11]. Although the restenosis rate in renal arteries is not as high as in small coronary vessels, it should be emphasized that if restenosis occurs, technical capabilities are limited. There are no available drug-eluting stents with a diameter over $5.0 \mathrm{~mm}$ dedicated to the renal arteries. Similarly, drug-eluting balloons designed for superficial femoral arteries are broad enough but too long to be used in renal arteries.

Obviously, the results of our study do not support performing CTA examination in every patient before renal artery stenting because of the additional radiation exposure and contrast-medium dose. However, in theory, it may be counterbalanced by the easier and safer renal artery cannulation (especially in the case of atypical take off or tight ostial stenosis), decreased risk of distal embo-
Table II. Characteristics of renal artery stenting procedure

\begin{tabular}{lc} 
Parameter & Result \\
\hline $\begin{array}{l}\text { Number of renal arteries } \\
\text { stented }\end{array}$ & 41 \\
\hline Predilatation, $n$ (\%) & $18(43.9)$ \\
\hline $\begin{array}{l}\text { Balloon for predilatation } \\
\text { diameter [mm] }\end{array}$ & $3.0(3.0-3.5)$ \\
\hline $\begin{array}{l}\text { Balloon for predilatation } \\
\text { length [mm] }\end{array}$ & $12(10-15)$ \\
\hline Stent diameter [mm] & $6.0(5.0-6.0)$ \\
\hline Stent length [mm] & $15(12-15.75)$ \\
\hline Residual stenosis (\%) & 3 (7.3) \\
\hline Postdilatation, $n$ (\%) & Herculink Elite (Abbott) 25 (61.0) \\
\hline $\begin{array}{l}\text { Name of stent } \\
\text { (manufacturer), } n(\%)\end{array}$ & Hippocampus (Invatec) 9 (22.0) \\
\cline { 2 - 2 } \begin{tabular}{l} 
Racer (Medtronic) 2 (4.9) \\
\cline { 2 - 2 }
\end{tabular} \\
\cline { 2 - 2 } & Prokinetic Energy (Biotronic) 2 (4.9) \\
\hline
\end{tabular}

Data presented as medians with interquartile range in brackets or numbers with percentage given in brackets.

lization (in the presence of aortic aneurysm, soft plaques or thrombi near the ostium) and less frequent need for additional postdilatation. Therefore, in selected patients with low risk of contrast-induced nephropathy preprocedural CTA may be considered. On the other hand, a majority of patients undergo CTA in the course of diagnostic process, before being referred for invasive treatment. Our study strongly suggests that careful evaluation of the available CTA scans by the operator before the invasive procedure may be beneficial and should be mandatory, provided that the pre-PTA CTA dataset is available.

Interestingly, the lesion length in CTA was only $1 \mathrm{~mm}$ longer than that measured in angiography CTA. In theory, CTA examination enables better identification of non-obstructive plaques as compared to conventional angiography. For that reason CTA may have potential advantages in stent length selection in terms of both better lesion coverage and lower probability of the "on the edge" restenosis. However, the renal artery plaques are rather non-diffuse, with typical ostial and proximal location. In the present population most of the plaques were shorter than $15 \mathrm{~mm}$, so there was no difference in lesion length or proposed stent length between the two imaging modalities. Surprisingly, the stents used in the procedure were $3 \mathrm{~mm}$ longer than retrospectively predicted in CTA and angiography (with a statistically significant difference between the angiography-derived proposal and the actual stent). The likely explanation is the intention of the operator to cover the whole plaque with broader healthy margins and to avoid uncovering the ostial lesions in case of the stent's distal protrusion, which may occur especially with implantation of short stents. 
The CTA-guiding strategy of stent sizing was investigated in patients with coronary artery disease. De Silva et al. evaluated 352 coronary lesions in 248 patients and demonstrated a significant correlation between CTA-proposed stent dimensions and the actual stent used [12]. The fact that the mean predicted stent length was slightly longer than the actual stent used confirms the hypothesis that coronary plaques are more diffuse, and therefore CTA may be a more reliable modality in their evaluation. However, in contrast to our report, the stent diameter chosen in CTA was smaller than that based on angiography.

Notably, in the report of LaBounty et al., the anticipated stent diameter and length were larger with CTA-based estimation than with angiography [13]. Similarly, in another randomized, single-center study, the stented segment length was longer and the nominal stent diameter tended to be larger in the computed tomography group [14]. Moreover, additional IVUS examination after stent implantation revealed that in the CTA-guided group, minimal stent area tended to be larger and the smallest peri-stent reference lumen area was significantly larger, with a smaller plaque burden as compared to angiography-guided patients. Pregowski et al. demonstrated in their study a larger reference segment diameter with CTA than with conventional angiography in saphenous vein graft lesions and suggested potential value of CTA in coronary intervention planning [15]. An additional trial assessing the utility of CTA in planning the strategy of bifurcation lesion angioplasty is ongoing [16].

Interestingly, the percent diameter stenosis estimation was significantly higher in angiography than in CTA. It should be taken into account that visual assessment of angiography results may overestimate the degree of the stenosis, leading in some cases to an unnecessary stenting procedure. It further supports the need for evaluation of the CTA results, if available, before the procedure.

There are a few limitations of our study. This is a single-center study with a relatively small number of patients. This is due to the decreasing number of renal artery stenting procedures performed in recent years and the large proportion of patients in whom CTA was not feasible (due to severe renal failure, bilateral renal stenosis or a strongly suggestive diagnosis of renal artery stenosis in Doppler duplex ultrasonography). Thus, the results should be interpreted with caution.

The study presents the results of a retrospective analysis, so we do not know in which cases preprocedural CTA analysis was actually performed and how it influenced the stent selection. On the other hand, considering that the selected stent diameter was identical to that proposed in angiography in $70 \%$ of cases, we can assume that the operator mostly relied on angiographic images.

\section{Conclusions}

Renal CTA has potential advantages as a tool adjunctive to angiography in appropriate stent sizing. Careful evaluation of the available CTA scans may be beneficial and so should be considered prior to planned renal stenting.

\section{Conflict of interest}

The authors declare no conflict of interest.

\section{References}

1. ESC guidelines on the diagnosis and treatment of peripheral artery diseases: document covering atherosclerotic disease of extracranial carotid and vertebral, mesenteric, renal, upper and lower extremity arteries. The Task Force on the Diagnosis and Treatment of Peripheral Artery Diseases of the European Society of Cardiology (ESC). Eur Heart J 2011; 32: 2851-906.

2. Fujihara M, Yokoi Y, Abe T, et al. J-RAS Study Investigators. Clinical outcome of renal artery stenting for hypertension and chronic kidney disease up to 12 months in the J-RAS Study prospective, single-arm, multicenter clinical study. Circ J 2015; 79: 351-9

3. Messerli FH, Bangalore S, Makani $\mathrm{H}$, et al. Flash pulmonary oedema and bilateral renal artery stenosis: the Pickering syndrome. Eur Heart J 2011; 32: 2231-5.

4. Kądziela J, Tyczyński P, Michałowska I, et al. Percutaneous angioplasty of the only patent renal artery - treatment of choice for end-stage heart failure. Postep Kardiol Inter 2013; 9: 83-5.

5. Wheatley K, Ives N, Gray R, et al. ASTRAL Investigators. N Engl J Med 2009; 361: 1953-62.

6. Cooper CJ, Murphy TP, Cutlip DE, et al.; CORAL Investigators. Stenting and medical therapy for atherosclerotic renal-artery stenosis. N Engl J Med 2014; 370: 13-22.

7. Murphy TP, Cooper CJ, Cutlip DE, et al. Roll-in experience from the Cardiovascular Outcomes with Renal Atherosclerotic Lesions (CORAL) study. J Vasc Interv Radiol 2014; 25: 511-20.

8. Fujii K, Carlier SG, Mintz GS, et al. Stent underexpansion and residual reference segment stenosis are related to stent thrombosis after sirolimus-eluting stent implantation: an intravascular ultrasound study. J Am Coll Cardiol 2005; 45: 995-8.

9. Cheneau E, Leborgne L, Mintz GS, et al. Predictors of subacute stent thrombosis: results of a systematic intravascular ultrasound study. Circulation 2003; 108: 43-7.

10. Kuntz RE, Gibson CM, Nobuyoshi M, et al. Generalized model of restenosis after conventional balloon angioplasty, stenting and directional atherectomy. J Am Coll Cardiol 1993; 21: 15-25.

11. Kastrati A, Schömig A, Elezi S, et al. Predictive factors of restenosis after coronary stent placement. J Am Coll Cardiol 1997; 30: 1428-36.

12. de Silva R, Mussap CJ, Hecht HS, et al. Stent sizing by coronary computed tomographic angiography: comparison with conventional coronary angiography in an experienced setting. Catheter Cardiovasc Interv 2011; 78: 755-63.

13. LaBounty T, Sundaram B, Chetcuti S, et al. Stent size selection using 64-detector coronary computed tomography angiography: a comparison to invasive coronary angiography. Acad Radiol 2008; 15: 820-6.

14. Pregowski J, Kepka C, Kruk M, et al. Comparison of usefulness of percutaneous coronary intervention guided by angiography plus computed tomography versus angiography alone using 
intravascular ultrasound end points. Am J Cardiol 2011; 108: 1728-34.

15. Pregowski J, Kepka C, Kalinczuk L, et al. Comparison of intravascular ultrasound, quantitative coronary angiography, and dual-source 64-slice computed tomography in the preprocedural assessment of significant saphenous vein graft lesions. Am J Cardiol 2011; 107: 1453-9.

16. Wolny R, Pręgowski J, Witkowski A. Coronary computed tomography angiography in planning of percutaneous coronary interventions in bifurcation lesions - study design and rationale. Postep Kardiol Inter 2013; 9: 155-9. 